\title{
Empreintes dues à l'implosion de bulles isolées : Etude paramétrique expérimentale
}

\author{
Dorey J. M., EDF, DER, Chatou \\ Nienaltowska E., ENSTA, Palaiseau
}

\section{Introduction}

Une quantification de l'agressivité de l'écoulement cavitant peut être réalisée au moyen d'échantillons polis placés à la paroi dans la zone soumise à l'érosion. La lecture des premières empreintes obtenues après un temps d'exposition relativement court aboutit à une "photographie filtrée " de l'agressivité. "Photographie » car chaque empreinte est la trace d'un impact sur le matériau. "Filtrée" car seuls les impacts ayant eu une puissance suffisante ont pu générer une empreinte. Cette puissance limite nécessaire est une caractéristique de l'état mécanique du matériau sous sa forme polie que l'on peut, pour simplifier, appeler seuil de marquage.

Une telle méthode de mesure d'agressivité érosive, proposée par Lecoffre [1], est un puissant moyen d'investigation (notamment pour la vérification de lois de similitude) si l'on peut établir un lien entre une grandeur hydrodynamique et l'échelle des seuils de marquage de divers matériaux. La pression du liquide lors du collapse est en toute logique l'élément déterminant de la violence des phénomènes. Elle s'impose donc comme paramètre de cette échelle. Comme procédure de correspondance entre pression et seuil, on propose d'utiliser les effets d'une bulle isolée créée par éclateur et implosant près du matériau à tester dans une cuve pressurisable.

\begin{abstract}
L'étude décrite ci-après a été entreprise dans l'optique de démontrer la faisabilité d'une telle procédure, et a eu pour objet de montrer l'influence des paramètres caractérisant le collapse à son origine (pression dans la cuve, rayon de la bulle et distance à la paroi) sur les empreintes obtenues sur deux matériaux: aluminium $99,99 \%$ et acier inoxydable $316 \mathrm{~L}$.
\end{abstract}

\section{Généralités}

\section{Taille et durée de vie de bulles}

Dans un milieu infini, une bulle créée par éclateur grossit puis implose sphériquement. Son temps de vie, $t_{b}^{*}$, peut être exprimé en fonction de son rayon maximum, $R_{\max }$, en utilisant la relation de Rayleigh [2]

$$
t_{b}^{*} / 2=0.915 R_{\max } \sqrt{\rho_{L}} /\left(P_{L}-P_{v}\right)
$$

où $P_{L}$ désigne la pression du liquide, $P_{v}$ la pression de saturation et $\rho_{L}$ la masse volumique du liquide.

Près d'une paroi solide, la bulle sera affectée dans sa phase de croissance mais surtout dans sa phase d'implosion. Dans ce cas, on définit un rayon équivalent, $R_{b}$, à partir du volume, $V$, de la bulle

\section{Impressions due to the implosion of isolated bubbles : experimental parametric study.}

With the aim of gaining a better understanding of the significance of various parameters on the cavitation erosion mechanism, an experimental study has been conducted on the marking of polished materials subjected to the collapse of a vapour bubble engendered by a discharger inside a pressurizable vessel. The parameters studied are : the pressure inside the vessel $P$, the distance relating to the centre of $a\left(d / R_{\max }<1.5\right)$ sample bubble, the material $(99.99 \%$ aluminium and $316 \mathrm{~L}$ stainless steel). Several conclusions can from now on be drawn :

* The implosion of a bubble can engender many holes, which demontrates that the collapse in the form of a torus plays a determining part.

* The maximum marking is obtained for a $d / R$ value of less than 1.1.

* For a given material, there exists a mximum permissible pressure below which no implosion is marked of about $0.07 \mathrm{MPa}$ for aluminium and $0.15 \mathrm{MPa}$ for steel. 

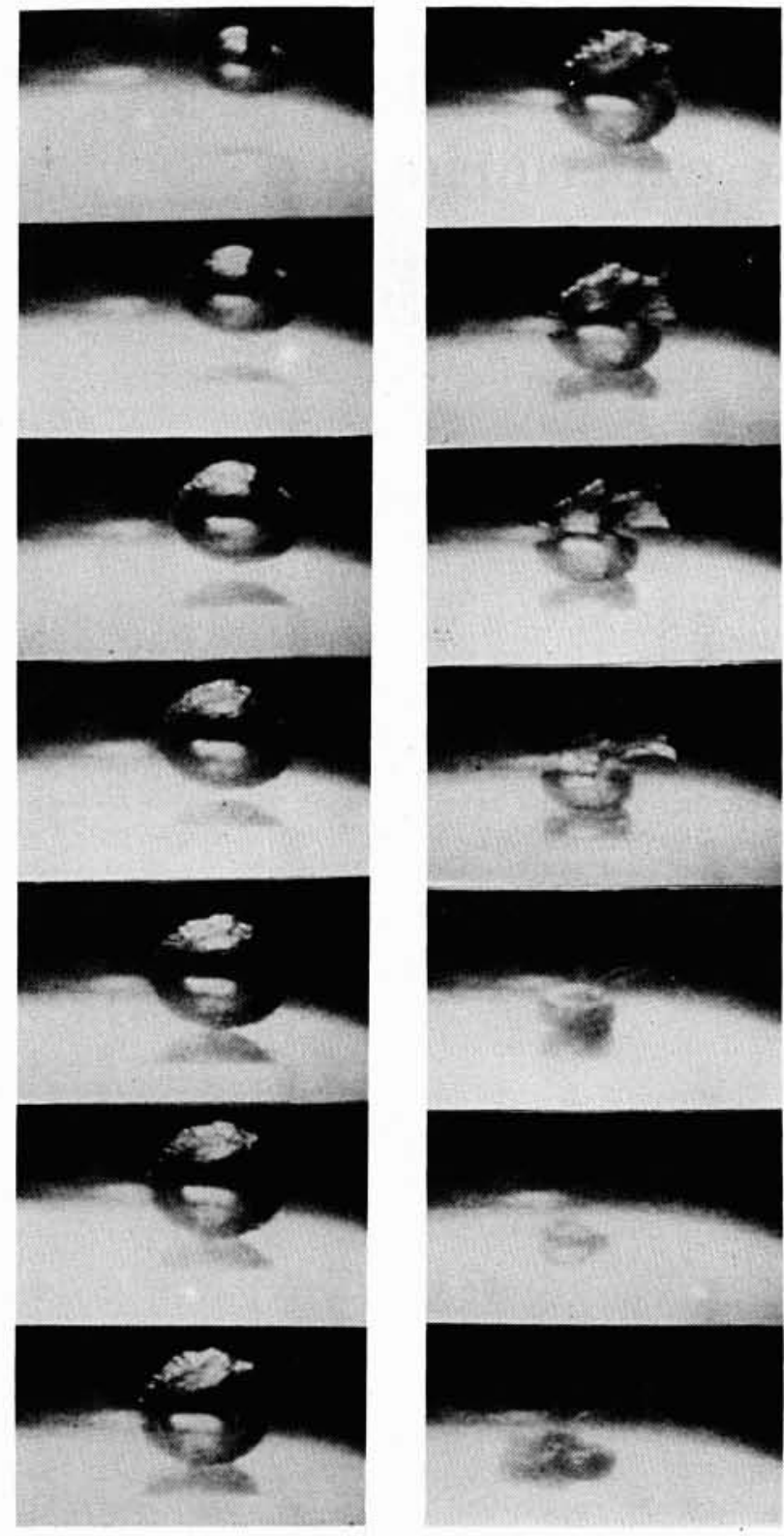

1. Implosion torique d'une bulle $\left(R_{\max }=6,5 \mathrm{~mm}\right)$ dans un écoulement, $V=5 \mathrm{~m} / \mathrm{s}$.

$$
R_{b}=(3 V / 4 \pi)^{1 / 3} .
$$

Il a été vérifié par cinématographie ultra-rapide que, pour une tension de décharge et une pression donnée, le volume de la bulle est indépendant de la distance $d$. Il en résulte que $\left(R_{b}\right)_{\max }=R_{\max }$. Par contre, la durée de vie, $t_{b}$, de la bulle est modifiée. Nous présentons dans le paragraphe consacré à la procédure expérimentale la façon dont varie $t_{b}$ et comment en déduire la valeur de $R_{\max }$.

\section{Phases d'implosion et influence de la pression}

L'asymétrie du flux de masse fluide lors du collapse, due à la présence de la paroi, se traduit par un mouvement plus rapide de la surface de la bulle opposée à la paroi formant un jet rentrant traversant la bulle. L'écoulement prend alors la forme d'un tourbillon torique organisé autour de la vapeur résiduelle qui continue son implosion. Pour une bulle créée au sein du liquide en écoulement (vitesse moyenne $5 \mathrm{~m} / \mathrm{s}$ ) cette évolution a pu être observée par cinématographie ultra-rapide, figure 1, où le temps séparant deux images est de $123 \mu \mathrm{s}$. La dépression locale, due à la présence du profil, a ralenti considérablement le mouvement. Dans la séquence présentée, la direction du jet rentrant par rapport à la paroi est légèrement affectée par le champ de vitesse autour de la bulle. On remarque que la fin du premier collapse (collapse du tore) est postérieure à l'impact du jet rentrant sur la paroi. L'observation des bulles de cavitation dans un liquide au repos [3], confirme la même chronologie dans l'évolution de bulles.

Lors de la première phase d'implosion, c'est-à-dire jusqu'au choc du microjet sur la paroi, l'hypothèse de similitude de l'écoulement (en dimension et en pression) est admissible. En effet, à ce stade les effets de compressibilité sont encore négligeables. On peut donc écrire que, si $V_{j}$ est la vitesse du microjet et $d_{j}$ son diamètre :

$$
V_{i} / \sqrt{\left(P_{L}-P_{v}\right) / \rho_{L}}=f\left(d / R_{\max }\right)
$$

et

$$
d_{j} / R_{\max }=g\left(d / R_{\max }\right) .
$$

Une approche monodimensionnelle du choc élastique du microjet contre la paroi opposée permet de donner l'ordre de grandeur de l'amplitude de l'onde de choc, selon la nature de la paroi

- dans le fluide :

$$
P_{0 L}=\rho_{L} c_{L} V_{j} / 2
$$

- dans le solide :

$$
P_{0 S}=\rho_{L} c_{L} V_{j} /\left(1+\rho_{L} c_{L} / \rho_{S} c_{S}\right)
$$

où $c$ est la célerité du son et l'indice $s$ représente le solide. Cependant, lors d'impact de gouttes sur une paroi solide [4] on a mesuré des pressions locales atteignant trois fois $\rho_{L} \quad c_{L} V_{j}$. Par ailleurs, des modélisations bidimensionnelles faisant intervenir l'angle au point de contact solide-liquide aboutissent à des valeurs analogues de pression maximale [5]. L'échelle de temps caractéristique de cette onde est liée à la taille du microjet :

$$
t_{0}=d_{j} / 2 c_{L} .
$$

Outre son effet direct sur le matériau, cette onde peut aussi influer sur le collapse du tore. Un autre phénomène qui peut jouer un rôle analogue sur le tore, dans le cas où la bulle est attachée à la paroi $\left(d / R_{\max }<1\right)$ est le choc entre le flux radial issu du jet et la paroi fluide qui lui fait face.

Ensuite, lors du rebond, la vapeur n'a plus une structure homogène mais présente l'aspect d'un nuage 
approximativement torique de petites bulles. Leur collapse collectif peut être l'occasion d'une amplification locale, les ondes de pression émises par l'implosion des unes forçant le mouvement de collapse des autres. Il a d'ailleurs été montré [6] que le second collapse générait, pour $d / R$ de l'ordre de 1 , une pression globale du même ordre que le premier, ce qui laisse supposer que localement au droit de petites bulles la pression émise puisse être plus importante.

Quelle que soit la ou les phases provoquant l'érosion la source qui conditionne la dynamique et donc la puissance des phénomènes est bien la pression du liquide.

\section{Déformation plastique du matériau}

L'apparition de la déformation rémanente de la surface du matériau résulte du dépassement de son seuil du comportement élastique, souvent donnée par le critère de Von Mises faisant intervenir la limite en cisaillement pur, $S_{0}$. Au-delà de cette limite, la déformation finale est déterminée par le comportement élastoplastique du matériau. Deux modélisations numériques récentes $[7,8]$ ayant pris en compte ce type de comportement ont mis en évidence les faits suivants :

i) une plastification en profondeur, sans déformation importante de la surface, pour des charges de l'ordre de $3 S_{0}$,

ii) une déformation significative de la surface pour des charges supérieures à $5 S_{0}$.

\section{Moyens et procédures expérimentaux}

Une chambre à bulles de 21 (fig. 2), en acier inoxydable et pouvant supporter des pressions allant de 0,01 à $0,081 \mathrm{MPa}$ absolu a été utilisée. Elle est munie de 3 hublots et de deux piquages permettant le remplissagevidange et le réglage de pression dans la cuve en la reliant soit au circuit de pressurisation soit à une pompe à vide. Dans sa paroi latérale sont montés face-à-face le porte-échantillon et le porte-électrode. Ce dernier se déplace sur une platine munie d'un vernier permettant le contrôle de la distance échantillon-électrode. L'électrode coaxiale est constituée d'un fil de tungstène (de $0,9 \mathrm{~mm}$ de diamètre) recouvert d'une gaine isolante et insérée dans un petit tube d'acier de $2,52 \mathrm{~mm}$ de diamètre extérieur. L'extrémité affûtée du fil dépasse d'un millimètre du tube.

Le générateur d'impulsions de haute tension comprend deux circuits. Un circuit primaire sert de relai au circuit principal qui fournit l'énergie de l'arc électrique formé aux extrémités d'électrodes immergées dans l'eau. Le circuit principal comprend 3 condensateurs de $0,047 \mu \mathrm{F}$, montées en parallèle. Leur tension de charge est réglable entre 4 et $10 \mathrm{kV}$, grâce à une alimentation extérieure au générateur. Les décharges électriques dans le circuit primaire sont déclenchées manuellement ou à l'aide d'un relai contrôlé par la caméra Hycam lors de la prise d'images.

L'échantillon métallique testé, de $3 \mathrm{~cm}$ de diamètre, vissé sur le porte-échantillon, peut subir une rotation

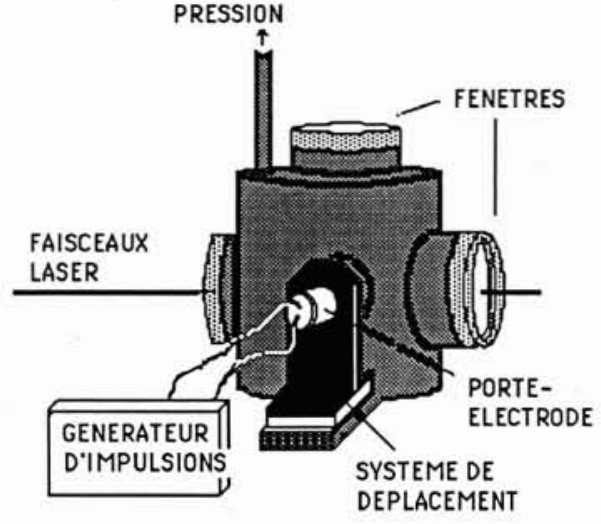

2. Schéma de la cuve pressurisable.

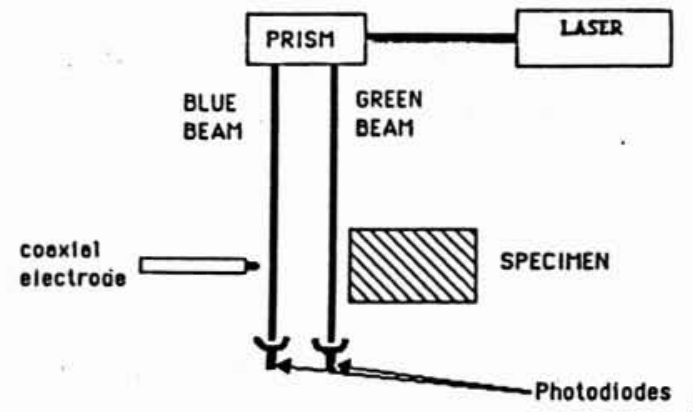

3. Schéma du dispositif laser pour la mesure de la durée de vie.

autour de son axe de façon à présenter devant l'électrode coaxiale différentes parties de sa surface. La préparation des échantillons d'aluminium a consisté en un polissage mécanique très fin, suivi d'un très court polissage électrolytique. La qualité de surface obtenue se caractérise par des dénivelés maximum inférieurs à $0,3 \mu \mathrm{m}$ pour des longueurs d'onde de l'ordre de $30 \mu \mathrm{m}$. Sur l'acier, le polissage mécanique a suffit à obtenir des dénivelés maximum de $0,02 \mu \mathrm{m}$ pour des longueurs d'onde de même ordre. Les profils d'empreintes ont été réalisés à l'aide d'un profilomètre type Talysurf 4 , d'une précision de $0,02 \mu \mathrm{m}$. Des reliefs ont été photographiés à l'aide d'un microscope équipé du contraste de Nomarsky.

Pour mesurer les durées de vie de bulles un dispositif optique a été mis en place. Deux faisceaux provenant d'un laser Argon (modèle $162 \mathrm{~A}$-Spectra Physics) sont focalisés au voisinage de l'échantillon métallique (fig. 3), l'un à proximité immédiate de la paroi (à $1 \mathrm{~mm}$ environ), l'autre plus près de l'électrode. L'intensité lumineuse de chaque faisceau est mesurée au moyen de photodiodes et enregistrée dans la mémoire d'un oscilloscope GOULD 4074. L'obturation ou l'atténuation partielle des faisceaux témoigne du passage d'une interface liquidevapeur ou d'une onde de choc. Ainsi, comme on peut le 


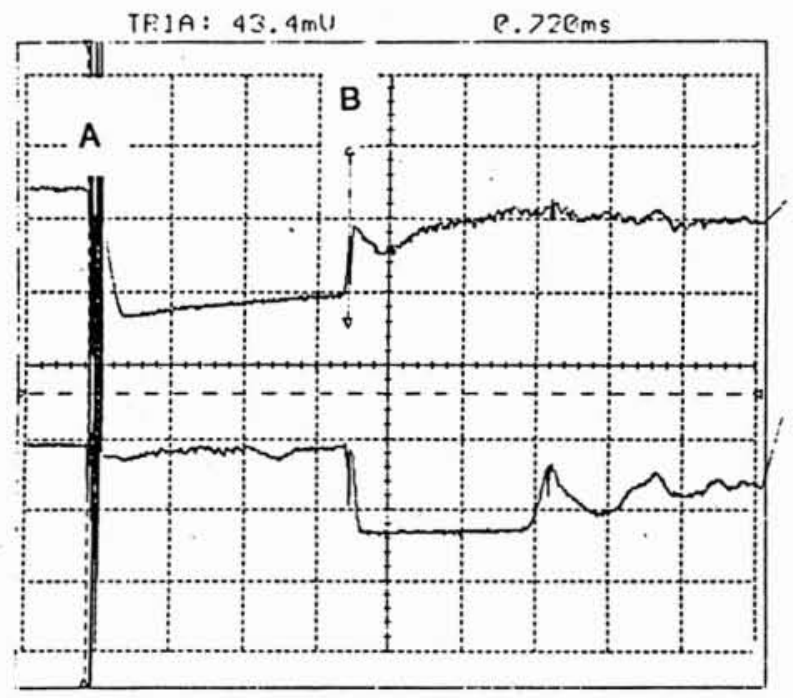

4. Réponse typique des faisceaux lors d'une implosion.

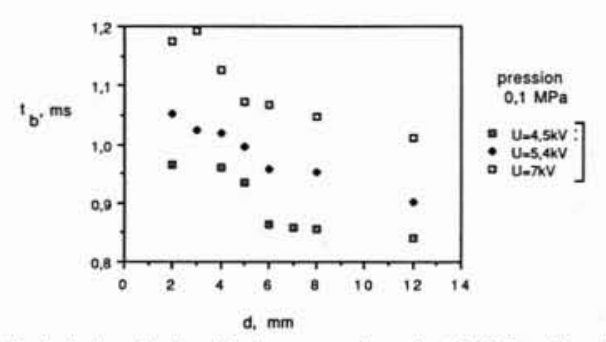

a) Durte de vie en fonction de la distance pour la pression de 0.1MPa et l'énergie de décharge variable

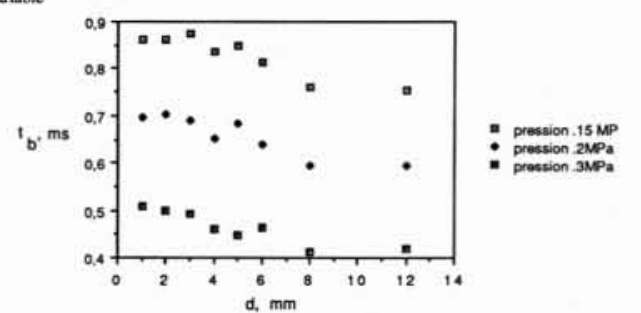

b) Durte de vie en fonction de la distance pour differents niveaux de pressions a l'énergie de decharge constante

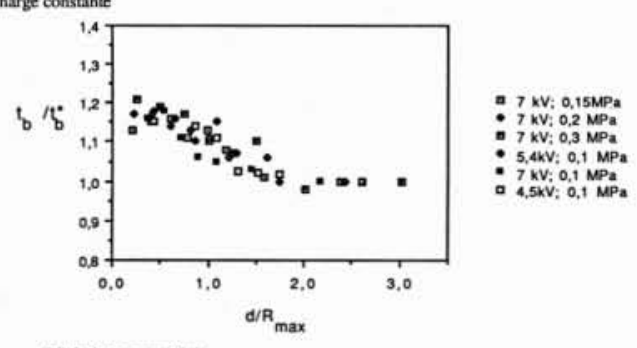

c) Resultats normalists

5. Influence de la proximité de la paroi sur les durées de vie de bulles.

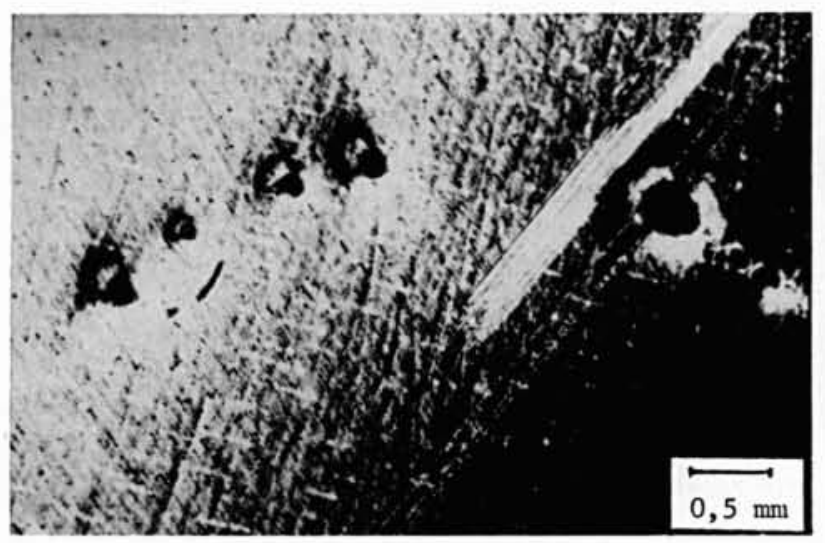

constater (fig. 4), sur le premier signal (faisceau plus proche de l'électrode), le grossissement commence dès l'apparition de l'étincelle, qui déclenche l'acquisition du signal. Le deuxième faisceau n'est interrompu que lors du passage de l'onde de choc et par le rebond. La durée de vie est mesurée entre l'étincelle et le passage de l'onde de choc avec une précision de $15 \mu \mathrm{s}$.

Pour obtenir la relation entre la durée de vie et le rayon critique de la bulle, nous avons cherché d'abord la variation de la durée de vie en fonction de la distance paroi-électrode. Des courbes obtenues pour différents niveaux de pression et d'énergie sont montrées dans la figure $5 a, b$. En prenant la durée de vie de la bulle en milieu « infini " comme l'échelle du temps et le rayon de la bulle lui correspondant comme l'échelle de longueur, toutes les courbes ont été adimensionnalisées et portées sur le même graphique, figure $5 c$.

Par conséquent, pour une distance et une pression données, la mesure du temps permet d'obtenir le rayon de la bulle. Le calcul est effectué par itérations.

\section{Résultats et discussion}

Les essais ont porté sur des bulles de rayon de 4,5 à $7 \mathrm{~mm}$, à des distances relatives de 0,6 à 1,5 . Les pressions appliquées ont été de 0,$05 ; 0,07 ; 0,1 ; 0,15$; 0,2 et $0,3 \mathrm{MPa}$. Les résultats sont présentés dans les tableaux 1 et 2 pour l'aluminium et l'acier respectivement. Compte tenu des limitations techniques nous n'avons relevé que les empreintes suffisamment larges. Le diamètre $d_{0,1}$ est le diamètre pour lequel le dénivelé atteint $10 \%$ de la profondeur maximale $h$. Les résultats pour des rayons inférieurs à $5 \mathrm{~mm}$ donnent moins de marquage. Des essais complémentaires sont en cours pour déterminer s'il s'agit d'un réel effet de taille. Dans l'immédiat les résultats obtenus pour $R_{\max }<5 \mathrm{~mm}$ ne seront pas pris en compte dans la discussion.

Pour des bulles de même rayon dans les mêmes conditions le nombre d'empreintes peut varier fortement et dans la plupart des cas elles sont décentrées par rapport à l'axe du premier collapse. Ce caractère aléatoire du marquage ainsi que la dispersion des valeurs de $d_{0,1}$ et $h$ a déjà été observée, à pression atmosphérique, par Lush et al. [9]. Dans certains essais sur l'aluminium, il a même été constaté que ces empreintes se répartissaient sur un arc de cercle (fig. 6), TOMITA \& SHIMA [10] ont relevé des distributions analogues sur de l'indium.

La dispersion en taille et nombre des empreintes ainsi que leur localisation confirment l'idée que l'érosion ne peut être attribuée à l'impact direct du microjet, phénomène bien localisé et répétitif. Elle est plutôt le résultat d'un état plus complexe où la multiplication des structures de vapeur est à l'origine d'une amplification locale par collapse collectif.

En dépit de ce caractère aléatoire, plusieurs faits ont été mis en évidence :

- Influence de la proximité de la paroi: A pression atmosphérique, les multiples essais effectués à $d / R>1,2$ n'ont donné aucune empreinte sur aluminium, alors que pour $0,6<d / R<1,1$ la probabilité de marquage est supérieure à 0,5 pour des bulles de 5 à $6 \mathrm{~mm}$.

6. Empreintes réparties sur un arc de cercle $\left(A_{1}\right)$. 
Tableau 1. Résultats pour aluminium 99,99\%.

\begin{tabular}{|c|c|c|c|c|c|c|}
\hline $\begin{array}{c}P_{L} \\
(M P a)\end{array}$ & $\begin{array}{c}R_{\max } \\
(\mathrm{mm})\end{array}$ & $d / R_{\max }$ & NOMBRE OEMPRENTES & $\begin{array}{c}h \\
(\mu m)\end{array}$ & $\begin{array}{l}d_{0,1} \\
(\mathrm{~mm})\end{array}$ & $n \boldsymbol{A d} 0.1$ \\
\hline 0.05 & & & $\begin{array}{l}\text { aucun marque quelque solt } \\
\text { io rayon ot la distance }\end{array}$ & & & \\
\hline \multirow[t]{6}{*}{0.1} & $\begin{array}{l}6.70 \\
6.38 \\
6.76 \\
6.32 \\
6.22 \\
6.44\end{array}$ & $\begin{array}{l}0.97 \\
1.02 \\
0.96 \\
0.63 \\
0.64 \\
0.62\end{array}$ & $\begin{array}{l}\text { une marque per bulle } \\
\text { +un chapelet do peitis trous dont } \\
\text { io plus important est } \\
\text { une merque par bulle }\end{array}$ & $\begin{array}{c}2.0 \\
6.5 \\
7.6 \\
16.0 \\
5.6 \\
12.5 \\
7.2\end{array}$ & $\begin{array}{l}1.7 \\
1.25 \\
0.5 \\
1.15 \\
0.7 \\
1.2\end{array}$ & $\begin{array}{l}0.001 \\
0.012 \\
0.032 \\
0.005 \\
0.018 \\
0.006\end{array}$ \\
\hline & $\begin{array}{l}6.10 \\
5.71 \\
5.93 \\
\end{array}$ & $\begin{array}{l}0.82 \\
0.70 \\
1.01 \\
\end{array}$ & une & 2.2 & 1.0 & 0.002 \\
\hline & $\begin{array}{l}5.9 \\
5.9 \\
\end{array}$ & $\begin{array}{l}1.1 \\
1.1 \\
\end{array}$ & une merque par bulle & $\begin{array}{l}7.4 \\
3.0 \\
\end{array}$ & $\begin{array}{l}0.85 \\
2.00\end{array}$ & \begin{tabular}{|l|}
0.009 \\
0.0015 \\
\end{tabular} \\
\hline & $\begin{array}{l}5.88 \\
6.1 \\
\end{array}$ & $\begin{array}{l}0.85 \\
0.82 \\
\end{array}$ & une & 4.0 & 200 & 0.002 \\
\hline & $\begin{array}{l}5.79 \\
5.93 \\
\end{array}$ & $\begin{array}{l}0.6 \\
0.59 \\
\end{array}$ & une & 5.6 & 1.05 & 0.005 \\
\hline & $\begin{array}{l}4.77 \\
5.27 \\
5.72 \\
5.10 \\
5.33 \\
5.40 \\
\end{array}$ & $\begin{array}{l}1.05 \\
0.95 \\
0.87 \\
0.59 \\
0.56 \\
0.56 \\
\end{array}$ & $\begin{array}{l}\text { une } \\
\text { wame }\end{array}$ & 1.8 & 0.85 & 0.002 \\
\hline \multirow[t]{2}{*}{0.15} & \begin{tabular}{|l|}
4.55 \\
4.55 \\
5.42 \\
5.28 \\
5.42 \\
\end{tabular} & $\begin{array}{l}1.05 \\
1.05 \\
1.07 \\
1.10 \\
1.07 \\
\end{array}$ & $\left\{\begin{array}{l}\text { une } \\
\text { une merque par bulle } \\
\text { deux }\end{array}\right.$ & $\begin{array}{l}11 \\
1.8 \\
15 \\
1.4 \\
\end{array}$ & $\begin{array}{l}1.1 \\
1.4 \\
0.8 \\
0.8 \\
\end{array}$ & $\begin{array}{l}0.010 \\
0.001 \\
0.019 \\
0.002 \\
\end{array}$ \\
\hline & \begin{tabular}{r|} 
de 4.1 \\
14.7 \\
\end{tabular} & $\begin{array}{l}\text { de } 1.0 \\
10.73 \\
\end{array}$ & pas dempreintes pour 6bulles & & & \\
\hline \multirow{4}{*}{0.2} & \begin{tabular}{|l|}
5.04 \\
5.88 \\
5.83 \\
\end{tabular} & \begin{tabular}{|l|}
0.93 \\
0.80 \\
0.80 \\
\end{tabular} & $\begin{array}{l}\text { marques dispostes en couronne } \\
\text { une }\end{array}$ & $\begin{array}{l}24 \\
15\end{array}$ & $\begin{array}{l}0.7 \\
1.2\end{array}$ & $\begin{array}{l}0.034 \\
0.0125\end{array}$ \\
\hline & $\begin{array}{l}5.04 \\
4.79 \\
4.89\end{array}$ & $\begin{array}{l}0.73 \\
0.81 \\
0.80\end{array}$ & quatre merque pour 3 bulles & $\begin{array}{l}4.2 \\
4.2 \\
8.2 \\
2.6 \\
\end{array}$ & $\begin{array}{l}0.85 \\
0.95 \\
0.8 \\
0.5 \\
\end{array}$ & $\begin{array}{l}0.005 \\
0.004 \\
0.010 \\
0.005 \\
\end{array}$ \\
\hline & $\begin{array}{l}5.17 \\
5.30 \\
\end{array}$ & $\begin{array}{l}1.24 \\
1.22 \\
\end{array}$ & une pour 3 bulles & 4.0 & 1.4 & 0.003 \\
\hline & $\begin{array}{l}5.4 \\
5.24 \\
4.95\end{array}$ & $\begin{array}{l}1.32 \\
1.36 \\
1.44\end{array}$ & wane & & & \\
\hline \multirow[b]{2}{*}{0.3} & $\begin{array}{l}4.19 \\
4.53 \\
\end{array}$ & $\begin{array}{l}1.05 \\
0.97 \\
\end{array}$ & une par bulle & $\begin{array}{l}1.8 \\
1.5 \\
\end{array}$ & $\begin{array}{l}1.5 \\
1.0 \\
\end{array}$ & $\begin{array}{l}0.0012 \\
0.0015 \\
\end{array}$ \\
\hline & $\begin{array}{l}4.60 \\
4.79 \\
4.79\end{array}$ & $\begin{array}{l}1.09 \\
1.04 \\
1.04\end{array}$ & $\begin{array}{l}\text { owcume } \\
\text { une }\end{array}$ & 7.8 & 0.8 & 0.010 \\
\hline
\end{tabular}

Tableau 2. Résultats pour acier $316 \mathrm{~L}$.

\begin{tabular}{|c|c|c|c|c|c|c|}
\hline $\begin{array}{c}P_{L} \\
\left(M P_{a}\right)\end{array}$ & $\begin{array}{r}R_{\max } \\
(m m)\end{array}$ & $d / R_{\max }$ & NOMBRE OEMPREINTES & $\begin{array}{c}\mathrm{h} \\
(\mu \mathrm{m})\end{array}$ & $\begin{array}{l}d_{0.1} \\
(\mathrm{~mm})\end{array}$ & $n / d_{0,1}$ \\
\hline 0.1 & & & wane & & & \\
\hline 0.15 & $\begin{array}{l}5,8 \\
5,9 \\
5.5\end{array}$ & $\begin{array}{l}0.86 \\
0.84 \\
0.91\end{array}$ & une pour 3 bulles & 0,97 & 0,3 & 0,0032 \\
\hline \multirow{3}{*}{0.2} & $\begin{array}{l}5,7 \\
5,8 \\
5,8\end{array}$ & $\begin{array}{l}1.03 \\
1,04 \\
1.04\end{array}$ & $\begin{array}{l}\text { deux } \\
\text { dimension de la plus grande }\end{array}$ & 0,64 & 0.26 & 0,0025 \\
\hline & $\begin{array}{l}5,1 \\
5,2\end{array}$ & $\begin{array}{l}0,97 \\
0,96\end{array}$ & une pour 2 bulles & 0,86 & 0,39 & 0,0022 \\
\hline & $\begin{array}{l}5.3 \\
5,0 \\
5,5 \\
\end{array}$ & $\begin{array}{l}0.75 \\
0.81 \\
0.73\end{array}$ & mane & & & \\
\hline 0.3 & $\begin{array}{l}5.7 \\
4.5\end{array}$ & $\begin{array}{l}0.79 \\
1.00\end{array}$ & mane & & & \\
\hline
\end{tabular}




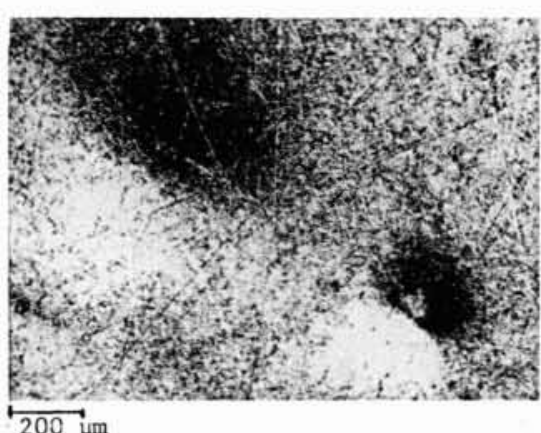

a)

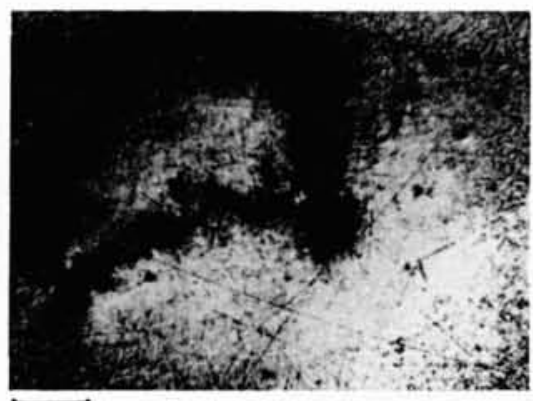

c)

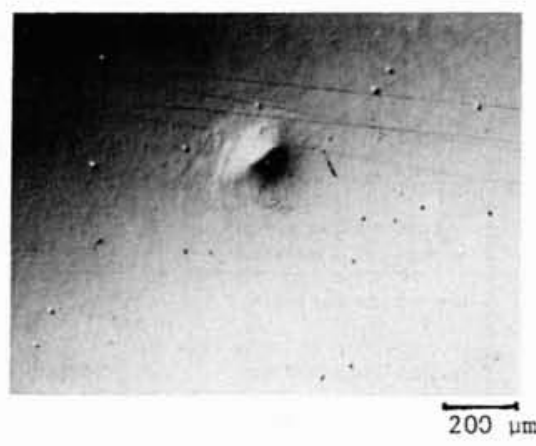

b)

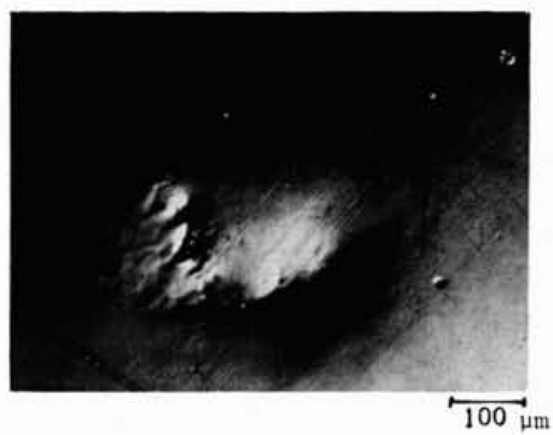

d)

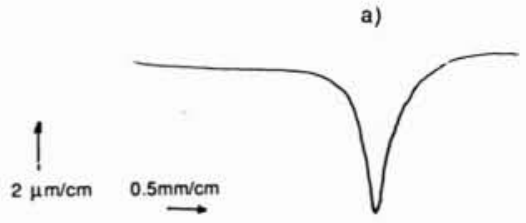

b)

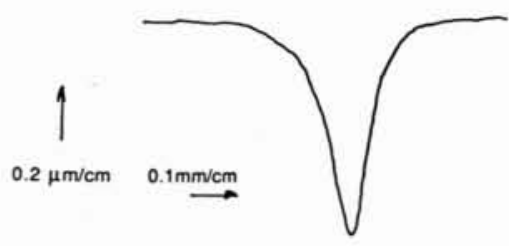

8. Profil type d'empreintes.

a) aluminium $99,99 \%$,

b) acier $316 \mathrm{~L}$.

7. Photographies d'empreintes. $a, c$ : aluminium; $b, d$ : acier $316 \mathrm{~L}$.

Par contre, aucune corrélation n'apparaît entre le rapport $h / d_{0,1}$ et le paramètre de proximité $d / R$.

- Influence de la pression: Sur l'aluminium, aucune marque n'a pu être produite pour plusieurs essais à 0,05 $\mathrm{MPa}$ alors qu'à $0,1 \mathrm{MPa}$ le taux de marquage est de l'ordre de $3 / 4$. Pour l'acier, les premières empreintes n'ont été obtenues qu'à une pression de $1,5 \mathrm{MPa}$.

- Formes des empreintes: Sur l'aluminium, les diamètres des plus grosses empreintes sont supérieurs à $1 \mathrm{~mm}$ alors que sur l'inox, ils sont inférieurs à $0,4 \mathrm{~mm}$. Les profondeurs peuvent atteindre $24 \mu \mathrm{m}$ sur l'aluminium alors qu'elles ne sont que de $1 \mu \mathrm{m}$ au maximum sur l'acier. Cependant malgré ces différences en tailles, les déformations présentent pour les deux matériaux les mêmes aspects: les marques peuvent être soit isolées (fig. $7 a, b$ ), et dans ce cas leur forme est symétrique, soit agglomérées ( $f i g .7 c, d$ ). La forme des profils relevés est caractéristique (fig. 8) : augmentation graduelle de pente sans rupture, ce qui dénote qu'il n'y a que déformation plastique, avec un point d'inflexion souvent proche du maximum. Les valeurs de $h / d_{0,1}$ varient entre 0,034 et 0,001 .

Sur l'acier inoxydable, la qualité du polissage a permis de détecter de toutes petites marques (fig. $7 a$ ), inférieures à $0,05 \mathrm{~mm}$ en diamètre, et assez nombreuses. Il est probable qu'elles soient le résultat de l'implosion de petites bulles au passage d'une onde de pression.

\section{Conclusions}

Pour des bulles créées par éclateur dans une cuve pressurisable, l'étude a abouti aux constatations suivantes :

1. Les plus grosses empreintes observées ont des diamètres de l'ordre de $1 \mathrm{~mm}$ sur l'aluminium et de l'ordre de $0,4 \mathrm{~mm}$ sur l'inox. Les profondeurs peuvent atteindre $24 \mu \mathrm{m}$ sur l'aluminium alors qu'elles ne sont que de $1 \mu \mathrm{m}$ au maximum sur l'acier.

2. La forme des profils relevés (augmentation graduelle de pente sans rupture), montre qu'il n'y a que déformation plastique du matériau.

3. A pression atmosphérique, le marquage ne peut être obtenu, sur l'aluminium, que pour des valeurs de $d / R$ inférieures à 1,2 .

4. Il existe pour chaque matériau, une valeur minimum de pression en deçà de laquelle la probabilité de marquer est faible : cette limite est de l'ordre de 0,07 MPa pour l'aluminium $99,99 \%$ et $0,15 \mathrm{MPa}$ pour l'acier $316 \mathrm{~L}$.

5. Compte tenu de la dispersion des empreintes, le marquage ne peut être le résultat direct du microjet.

La procédure proposée paraît adaptée à la caractérisation du seuil de marquage de matériaux, sous réserve d'effectuer un nombre suffisant d'essais pour que l'aspect statistique soit intégré. 


\title{
Références
}

[1] LeCoffre, Y. (1983): Erosion de cavitation. Similitudes hydrodynamiques. Journée Erosion de Cavitation. Grenoble, 30 mars 1983.

[2] Chahine, G. L. (1979): Etude locale du phénomène de cavitation. Analyse des facteurs régissant la dynamique des interfaces, thèse de doctorat d'état, Université Paris VI.

[3] Shutler, N. D., Mesler, R. B. (1965): A photographic study of dynamics and damage capabilities of bubbles collapsing near solid boundaries. J. Basic Engng, june 1965, pp. 511-517.

[4] Rochester, M. C., Brunton, J. H., Surface pressure distribution during drop impingement, 4 th Int. Conf. on Rain Erosion and Related Phenomena, Meersburg, RFA, 1974.

[5] Rosenblatt, M., Ito, M. Y., Eggum, G. E., Analysis of Brittle Target Fracture from a subsonic water drop impact, Erosion : Prevention and Useful Applications, ASTM STP 664, Ed. Adler, W. F., 1979, pp. 227-254.

[6] Shima, A., Takayama, K., Tomita, Y. (1984): Mechanism of the bubble collapse near a solid wall and induced impact pressure generation, Rep. Inst. High Speed Mech., vol. 48, n`367, Tohoku University, Sendai, Japan.

[7] Reboud, J. L., Réponse impulsionnelle d'un milieu élastoplastique. Application à l'étude de l'érosion de cavitation. Thèse de doctorat présenté à l'Université de Grenoble, 1987.

[8] CURNIER, A., RIDAh, S., Impact of a liquid microjet on an elastic-plastic solid surface, Proc. 7th Int. Conf. on Erosion by Liquid and Solid Impact, Cambridge, 1987.

[9] Lush, P. A., Wood, R. G. K., Carpanini, L. J. (1985) : Pitting in soft aluminium produced by spark-induced cavitation bubbles. Proceedings of VI Int. Conf. on Erosion by Liquid and Solid Impact.

[10] TомiтA, Y., Shima, A. (1986) : Mechanism of impulsive pressure generation and damage pit formation by bubble collapse, J. of Fluid Mechanics, vol. 169, pp. 535-564.

\author{
Adresses des auteurs : \\ Monsieur J. M. Dorey \\ EDF-DER \\ 6, quai Watier \\ 78000 Chatou \\ Tél. : $307177 \quad 17$ \\ Madame E. Niewaltowska \\ ENTA-Groupe Phénomènes d'interface \\ Chemin de la Hunière \\ 91000 Palaiseau \\ Tél. : 60100318
}


M. GRISON : Je voudrais apporter un commentaire sur cette étude et la resituer dans la démarche de la recherche d'une similitude de l'érosion de cavitation dont parlera M. AMBLARD du CERG. Il s'agissait de vérifier que la capacité de marquer un matériau donné dépendait surtout de la pression existant à l'endroit où implose la bulle et non de la taille de la bulle (pour des positions relatives de la bulle constante). C'est bien ce qui a été trouvé et ce qui confirme que cette faculté de marquage est indépendante de l'effet d'échelle si l'on respecte les lois de pressions, ce qui avait été trouvé par M. LECOFFRE.

Un deuxième point important est qu'il est très difficile de passer de manière théorique de l'hydrodynamique d'une bulle isolée à l'empreinte dans le matériau. Cette démarche sera a fortiori plus difficile dans l'écoulement et justifie pleinement la démarche consistant à révéler le "potentiel érosif " d'une configuration cavitante par une lecture des déformations à la surface d'un échantillon.

M. CHINCHOLLE informe l'assistance qu'au Laboratoire de Génie Electrique sont effectués depuis 15 ans des travaux sur l'usinage par électro-érosion. Ces travaux peuvent aider à mieux comprendre le mécanisme d'implosion d'une bulle provoquant arrachement de matière, cette bulle étant créée à partir d'électrodes distantes de $100 \mu$.

\section{CANAVELIS}

$I^{\text {re }}$ question: Pourquoi déterminez-vous le rayon de la bulle à partir du temps de Rayleigh, plutôt qu'à partir d'une visualisation directe puisque vous avez des moyens photographiques ou cinématographiques?

$2^{e}$ question : Avez-vous pu expérimentalement estimer l'ordre de grandeur des pressions d'impact, soit à l'aide de mini-capteurs, soit par photoélasticimétrie?

$3^{e}$ question: Vous trouvez qu'il y a un niveau de pression en dessous duquel il n'y a pas forcément déformation de surface. Mais peut être y a-t-il déjà une atteinte du réseau cristallin en dessous de la surface auquel cas la visualisation des impacts n'est peut être pas suffisante pour voir comment évoluera l'érosion.

MIIE NIENALTOWSKA :

1) Compte tenu du nombre important d'essais (environ 20 échantillons et de 4 à 6 impacts par échantillon), on ne peut envisager de filmer systématiquement chaque bulle pour des raisons de coût et de temps (dépouillement).

La détermination de la taille des bulles à l'aide d'une mesure optique, en utilisant le calibrage obtenu au préalable, est un moyen très efficace qui est plus précis que le relevé des contours sur les images filmées en raison de la définition imprécise (contraste) de celles-ci.

2) Nous avons comparé, en opérant dans une cuve spécialement conçue pour les mesures acoustiques, les signaux obtenus par photoélasticimétrie au sein du matériau et les signaux acoustiques. Au cours du premier collapse les deux types de signaux sont comparables. Au rebond et pendant les collapses successifs les signaux de pression ne sont plus représentatifs du niveau de pression exercée sur le matériau. Notons que la mesure par photoélasticimétrie donne une idée assez précise de la pression transmise au matériau, mais seulement à une profondeur de l'ordre de quelques millimètres de la surface.

3) En ce qui concerne l'endommagement de la structure cristalline, nous n'avons procédé à aucun examen qui permettrait d'en juger.

M. DOREY: Pour répondre à la troisième question de M. CANAVElis, je rappelle que le but de cette manipulation est de déterminer le seuil de marquage d'un matériau et non pas sa capacité de résister à l'érosion.

\section{CANAVELIS}

$4^{e}$ question: On a dit que le rayon de la bulle n'intervenait pas tellement sur la pression environnante au moment du collapse. Existe-t-il une tendance montrant que la loi de Rayleigh n'est pas vérifiée?

\section{Mme NIENALTOWSKA}

4) Il s'avère que des bulles de petite taille formées pendant le rebond, dites de la "deuxième génération ", marquent également le matériau. Cependant leur distance à la paroi n'est pas bien connue, par conséquent on ne peut donc fournir leur paramètre de proximité. Il serait plus approprié de parler de marquages dus aux implosions des structures de vapeur (multitude des petites bulles) que de l'implosion d'une bulle.

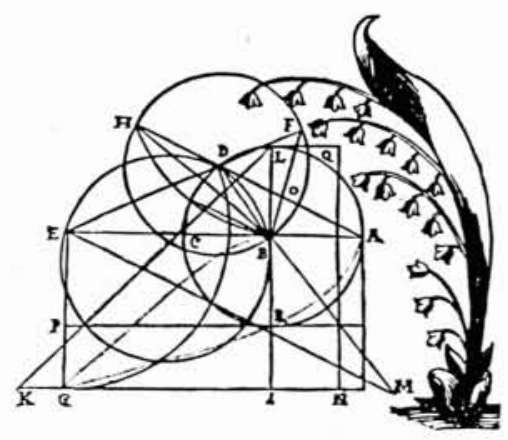

